\title{
Simulations of fast crab cavity failures in the high luminosity Large Hadron Collider
}

\author{
Bruce Yee-Rendon ${ }^{*}{ }^{\dagger}$ and Ricardo Lopez-Fernandez \\ Departamento de Física, Centro de Investigación y de Estudios Avanzados del I.P.N., \\ Apartado Postal 14-740, 07000, México, D.F. Mexico
}

Javier Barranco, ${ }^{\ddagger}$ Rama Calaga, Aurelien Marsili, Rogelio Tomás, and Frank Zimmermann European Organization for Nuclear Research (CERN), CH-1211 Geneva 23, Switzerland

Frédéric Bouly

LPSC, Université Joseph Fourier Grenoble, CNRS/IN2P3, Grenoble INP, Grenoble, France

(Received 16 December 2013; published 6 May 2014)

\begin{abstract}
Crab cavities (CCs) are a key ingredient of the high luminosity Large Hadron Collider (HL-LHC) project for increasing the luminosity of the LHC. At KEKB, CCs have exhibited abrupt changes of phase and voltage during a time period of the order of a few LHC turns and considering the significant stored energy in the HL-LHC beam, CC failures represent a serious threat in regard to LHC machine protection. In this paper, we discuss the effect of $\mathrm{CC}$ voltage or phase changes on a time interval similar to, or longer than, the one needed to dump the beam. The simulations assume a quasistationary-state distribution to assess the particles losses for the HL-LHC. These distributions produce beam losses below the safe operation threshold for Gaussian tails, while, for non-Gaussian tails are on the same order of the limit. Additionally, some mitigation strategies are studied for reducing the damage caused by the $\mathrm{CC}$ failures.
\end{abstract}

DOI: 10.1103/PhysRevSTAB.17.051001

PACS numbers: 29.20.db

\section{INTRODUCTION}

The high luminosity LHC (HL-LHC) upgrade program aims to use crab cavities (CCs) together with the reduction of the beam sizes at ATLAS and CMS interaction points (IP) and an increase of the beam intensity to enhance the integrated luminosity per year by up to a factor of 10 with respect to the nominal LHC of $10^{34} \mathrm{~cm}^{-2} \mathrm{~s}^{-1}[1,2]$. The relevant optics parameters for the HL-LHC scenario are summarized in Table I.

The CCs produce a transverse rotation on the bunches to recover the luminosity loss due to the crossing angle scheme at the IP [3,4]. After the installation in 2007, CCs have played an important role in reaching the luminosity record at the KEKB $\mathrm{e}^{+} \mathrm{e}^{-}$collider [5]. The HL-LHC will be the first hadron collider to operate with CCs.

At $\mathrm{KEKB}$, one $\mathrm{CC}$ was installed per beam, one at the high-energy ring (HER) and one at the low-energy ring

\footnotetext{
byee@fis.cinvestav.mx bruce.yee.rendon@cern.ch

Also at European Organization for Nuclear Research (CERN), CH-1211 Geneva 23, Switzerland.

Present address: Ecole Polytechnique Fédérale de Lausanne (EPFL), CH-1015 Lausanne, Switzerland.

Published by the American Physical Society under the terms of the Creative Commons Attribution 3.0 License. Further distribution of this work must maintain attribution to the author(s) and the published article's title, journal citation, and DOI.
}

(LER). During KEKB CC operation, from 13th February 2007 to 24th December 2009, some abrupt failures were observed in which the phase changed by $\pm 50^{\circ}$ within $50 \mu \mathrm{s}$ and the voltage dropped to zero within $100 \mu \mathrm{s}$, for a natural time constant of the CC $\left(\tau_{\text {crab }}=2 \mathrm{Q}_{\mathrm{L}} / \omega\right)$ of $84 \mu$ s for HER and $130 \mu$ s for LER [6]. The main causes of the CC failures at HER were breakdowns of superconductivity due to discharge in the cavity and for the CC at LER discharges in the coaxial and input couplers. The average of failures were 1.3 perl day at HER and 0.5 per day at LER [7]. Although the CC designs for the HL-LHC (compact CCs [8]) are different than the KEKB (elliptical), similar failures as at KEKB can occur at the HL-LHC compromising the machine protection. Indeed, if an abnormal beam behavior is detected at the LHC, the beam interlock system and the

TABLE I. Relevant optics parameters of the HL-LHC.

\begin{tabular}{lcc}
\hline \hline Parameter & Symbol & Value \\
\hline Energy & $E[\mathrm{TeV}]$ & 7 \\
Protons/bunch & $N_{b}\left[10^{11}\right]$ & 2.2 \\
Bunches & $n$ & 2808 \\
rms bunch length & $\sigma_{z}[\mathrm{~cm}]$ & 7.55 \\
Beta function at IP1,5 & $\beta^{*}[\mathrm{~m}]$ & 0.15 \\
Normalized emittance & $\epsilon[\mu \mathrm{m}]$ & 3.75 \\
Full crossing angle & $\phi[\mu \mathrm{rad}]$ & 590 \\
Main/crab RF & $\omega[\mathrm{MHz}]$ & 400 \\
Revolution period & $\tau[\mu \mathrm{s}]$ & 89 \\
Synchrotron period & $\tau_{s}[\mathrm{LHC}$ turns $]$ & 562 \\
\hline \hline
\end{tabular}


LHC beam dumping system can take up to 3 LHC turns (about $300 \mu \mathrm{s}$ ) to extract the full beam $[9,10]$. CCs prototypes will first be tested in the SPS early in 2017 [11].

At $7 \mathrm{TeV}$, the energy stored in a full HL-LHC beam of 2808 bunches is $692 \mathrm{MJ}$. A fast impact of $3 \%$ of a single bunch $(\sim 7 \mathrm{~kJ})$ is beyond the damage threshold of the superconducting magnets and a fraction about $10^{-8}(\sim 3 \mathrm{~J})$ of the full beam could already quench a magnet [12]. A main dipole or quadrupole will quench if $10-20 \mathrm{~mJ} / \mathrm{cm}^{3}$ is deposited in the coil [13].

To quantify the risk and to explore possible mitigation techniques, an extensive comparison of beam loss simulations for LHC and HL-LHC has been performed for fast CCs failures [14-17].

The CCs apply a transverse angular kick dependent on longitudinal position on the particle in the bunch. The kick applied is represented as

$$
\Delta p_{t}=-\frac{q \cdot V \cdot \sin \left(\phi_{s}+\frac{\omega z}{c}\right)}{E},
$$

where $\Delta p_{t}$ is the transverse angular kick, $q$ the particle charge, $V$ the CC voltage, $\phi_{s}$ the phase of the CC cavity with respect to the synchronous particle, $\omega$ the angular RF frequency of the $\mathrm{CC}, z$ the longitudinal position of the particle with respect to the bunch center, $c$ the speed of light and $E$ the particle energy.

In the local CC scheme a pair of groups of CCs per beam is placed on either side of the IP with the phase advance between the CC and the IP optimized to be $\pi / 2$ [18]. The total effect of a failure in this scheme is reduced by installing groups of CCs instead of a single CC. The CC voltage required to open the crab bump in order to produce an effective head-on collision is given by

$$
V_{1}=\frac{c \cdot E \cdot \tan \left(\frac{\phi}{2}\right)}{q \cdot \omega \cdot \sqrt{\beta_{\mathrm{IP}} \cdot \beta_{\mathrm{CC}}} \cdot \sin (\Delta \psi) \cdot n_{\mathrm{cc}}},
$$

and the voltage to close the bump is

$$
V_{2}=-M_{22} \cdot V_{1},
$$

where $V_{1}$ denotes the voltage of the CCs that opens the crab bump, $c$ the speed of light, $E$ the particle energy, $\phi$ the full crossing angle at IP, $q$ the particle charge, $\omega$ the RF frequency of the $\mathrm{CCs}, \beta_{\mathrm{IP}}$ the beta function at the interaction point, $\beta_{\mathrm{CC}}$ the beta function at the $\mathrm{CC}$ that opens the crab bump, $\Delta \psi$ the difference of phase advance between the IP and the CCs $(\sim \pi / 2), V_{2}$ the voltage of the CCs that closes the crab bump, $M_{22}$ is the $(2,2)$ element of the transfer matrix from the CC that opens the bump to the one which closes it, and $n_{\mathrm{cc}}$ the number of CCs per beam on both sides of the IP [18].

The local CC scheme allows flexibility in the optics and crossing scheme, however, due to the limited distance
TABLE II. CCs parameters for the HL-LHC. Subscript 1 refers to the CCs that open the crab bump and subscript 2 refers to the CCs which close it.

\begin{tabular}{lcc}
\hline \hline Parameter & Symbol & IP (ATLAS, CMS) \\
\hline $\mathrm{CC}_{1}$ voltage & $V_{1}[\mathrm{MV}]$ & 3.5 \\
$\mathrm{CC}_{2}$ voltage & $V_{2}[\mathrm{MV}]$ & 3.8 \\
Beta function at the $\mathrm{CC}_{1}$ & $\beta_{1}[\mathrm{~m}]$ & $3470-3652$ \\
Beta function at the $\mathrm{CC}_{2}$ & $\beta_{2}[\mathrm{~m}]$ & $2795-3290$ \\
Dispersion at the $\mathrm{CC}_{1}$ & $D_{1}[\mathrm{~m}]$ & 0.09 \\
Dispersion at the $\mathrm{CC}_{2}$ & $D_{2}[\mathrm{~m}]$ & -0.09 \\
\hline \hline
\end{tabular}

$(19 \mathrm{~cm})$ between the two proton beams, tight aperture constraints are imposed. To this end, compact cavity designs at lower frequency were developed to have a transverse footprint compatible with the LHC constraints [19]. The CCs scheme considered in the present study consists of three CCs per beam on either side of IP5 (CMS) and IP1 (ATLAS) and their parameters are described in Table II.

\section{SIMULATION SETUP}

In order to determine the CC effects in the LHC machine protection performance, the amount and positions of the losses are recorded in the cases when they work normally and when they present a failure. To this end, CC simulations for LHC and HL-LHC were done with the help of computer programs MAD-X [20] and SiXTRACK [21].

MAD-X allows us to design particle accelerator lattices, to simulate the beam dynamics and to improve the optics parameters. The local CC scheme was implemented in the lattice and the $\mathrm{CC}$ voltages calculated by MAD-X. The CC effects on the horizontal orbit $\mathrm{X}$ in MAD-X when the phase of the $\mathrm{CCs}$ is $0^{\circ}$ or $90^{\circ}$ are shown in Fig. 1.

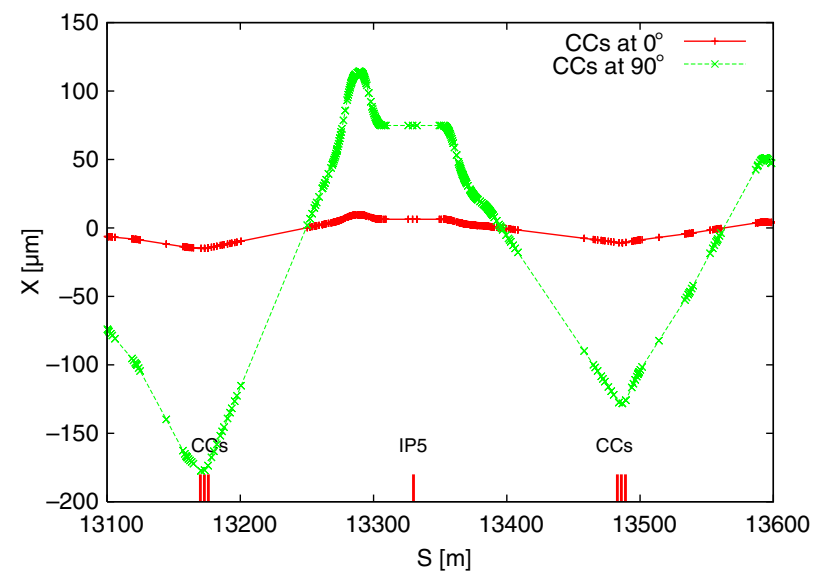

FIG. 1. Effect of the CCs on the X orbit of a particle at $0.133 \sigma_{z}$ $\left(1 \sigma_{z}=75.5 \mathrm{~mm}\right.$ ) longitudinal position when the CC phases are $0^{\circ}$ and $90^{\circ}$ around IP5. According to Eq. (1) the kick is small when the CC phase is $0^{\circ}$ (red) and becomes significant close to $9 \sigma_{x}\left(1 \sigma_{x}=8.7 \mu \mathrm{m}\right)$ at IP when the CC phase is $90^{\circ}$ (green). 


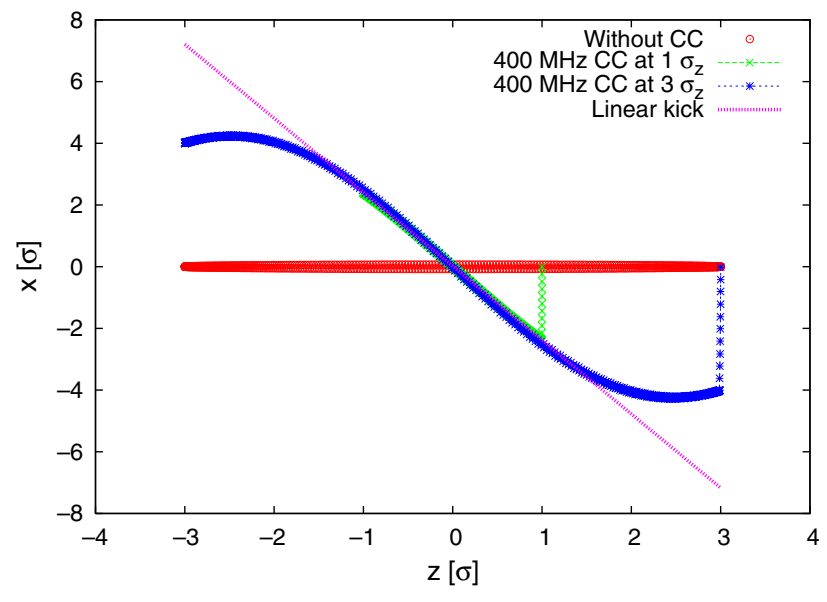

FIG. 2. Effect of the CCs turn by turn on the X orbit of a particle at different longitudinal positions from the center of the bunch at IP5 during 600 LHC turns (about 1 synchrotron period). These $\mathrm{CC}$ effects are compared with the ideal linear kick.

SIXTRACK 6D simulations are used for long term stability studies (dynamic aperture, collimation, tune optimization, etc.) in the LHC $[18,22]$. A modified version of SixTRACK was employed to introduce multiple CCs and to manipulate their parameters (voltage and phase). The turn by turn $\mathrm{CC}$ effect on individual particles was simulated at different longitudinal positions by tracking over 600 LHC turns (about 1 synchrotron period) with SIXTRACK. These effects are compared to the cases without $\mathrm{CC}$ and with an ideal linear kick (Fig. 2). For the case without CC, the X orbit remains undisturbed. In the cases of CC, two vertical lines can be seen at $1 \sigma_{z}$ and $3 \sigma_{z}$ corresponding to the ramping of the CC voltage (over $10 \mathrm{LHC}$ turns to simulate an adiabatic ramping). The kick received for a particle at $1 \sigma_{z}$ is in the linear range. On the other hand, for a particle at $3 \sigma_{z}$ a sinusoidal effect of the CC can be seen.

\section{A. Collimation system}

The collimation system is designed to protect the LHC components against unwanted beam losses. The collimators remove the particles beyond the safety range specified for the transverse beam size which could eventually impact on the beam pipes or magnets. An excellent level of cleaning efficiency is needed in order to avoid quenches of the superconducting magnets. The global inefficiency $(\eta)$ of the collimation system which is the particles that impact in the aperture divided by the total impacts on the machine, defines its performance. The collimation system in the LHC is mainly located at Interaction Region 3 (IR3) known as "momentum cleaning" and Interaction Region 7 (IR7) known as "betatron cleaning." The collimators can be classified in: primary (TCP), secondary (TCSG), absorbers for showers in cleaning insertions (TCLA), absorbers for physics debris (TCLP), injection protection (TCLI, TDI), beam dump (TCDQ, TCSTCDQ), and tertiary (TCT). The settings of the collimators are shown in Table III.
TABLE III. The collimator settings used in the CC study for the HL-LHC.

\begin{tabular}{lc}
\hline \hline Collimator & Nominal opening $[\sigma]$ \\
\hline TCP IR7 & 6 \\
TCSG IR7 & 7 \\
TCLA IR7 & 10 \\
TCP IR3 & 12 \\
TCSG IR3 & 15.6 \\
TCLA IR3 & 17.6 \\
TCLP & 12 \\
TCLI & 10 \\
TCSTCDQ IR6 & 7.5 \\
TCDQ IR6 & 8 \\
TDI & Open \\
TCT IR1/IR5 & 8.3 \\
TCT IR2/IR8 & 30 \\
\hline \hline
\end{tabular}

In the case of a CC failure, the crab bump is uncompensated, producing an oscillation of part of the beam which can lead to an increase in the beam losses. When a failure is detected, the LHC beam interlock system dumps the complete beam in about 3 LHC turns. During this time the collimator system represents the only passive protection for the LHC components. For this reason, full collimation studies are performed to assess the damage produced by a $\mathrm{CC}$ failure. The beam losses can be classified in two categories; namely impacts on the collimators or on the machine aperture. The first type of impacts refers to the particles which have an inelastic scattering with the collimators, mainly the TCPs and TCSGs. The second refers to the particles which have elastic scattering in collimators and then impact on the elements of the machine (cold magnets, warm magnets, etc.). Most of the tools necessary for this study were implemented by the collimation team in the SiXTRACK code [23].

\section{B. Beam distributions}

The standard LHC collimation studies track halo distributions to evaluate the beam losses [23]. Usually for LHC, a thin halo with a smear of $0.015 \sigma$ is generated in the horizontal phase space at a certain transverse position near the location of the primary collimators. Due to nonlinearities in the LHC, the simulated average impact parameter is about $0.15 \sigma$ (40 $\mu \mathrm{m}$ at the primary collimators). This value is higher than the one deduced in Ref. [24] from measured halo diffusion coefficients (between $0.02 \mu \mathrm{m}$ and $0.3 \mu \mathrm{m}$, depending on betatron amplitude and beam conditions). Nevertheless, the efficiency of the collimation system remains approximately the same and it is more efficient from the computing time point of view. For the vertical phase space a simple Gaussian distribution is used and, optionally also for the longitudinal beam distribution.

In this study, we use 2D Gaussian with matching beam conditions to generate the beam distribution in the phase 
space for both transverse and longitudinal planes. Due to low statistics in the tails, where particles are more likely to hit the aperture and to avoid CPU limitations, we split the transverse distribution into core and tail in the phase space. Taking into account that the maximal displacement produced by the failures of one $\mathrm{CC}$ in previous studies was $2.1 \sigma$ [16], we generated the core from 0 to $3 \sigma$ and the tails from $3 \sigma$ to $5.5 \sigma$. For the longitudinal distribution we generated the complete Gaussian distribution. Using these guidelines different simple Gaussians (SG) are produced:

(i) SG I: The beam cores were simulated generating the transverse phase space distributions up to $3 \sigma$, which corresponds to the $97.8 \%$ of the full beam.

(ii) SG II: To increase statistics in the tails the particles below $3 \sigma$ were removed in both horizontal and vertical phase space. The remaining distribution represents $0.012 \%$ of the full beam. (iii) SG III: In this case the particles below $3 \sigma$ in the horizontal phase space distribution were removed keeping the tails and on the vertical phase space only the core was kept. This corresponds to $1.1 \%$ of the full beam.

(iv) SG IV: It is the complementary case of SG III, the tails in the vertical phase space were kept and the core in the horizontal phase space distribution. This represents $1.1 \%$ of the entire beam.

In addition, more realistic beam distributions are obtained in two different ways; namely either by using the parameters from Van der Meer scans on CMS [25] or by applying the Abel transformation [26] to scraping measurements of the LHC beam at injection energy of $450 \mathrm{GeV}$ (Fig. 3). The distributions generated correspond to double Gaussians (DG):

(i) DG I: Van der Meer scan calibrates the luminosity measurement. This method scans beam profiles by separating the beams in the transverse plane [27].
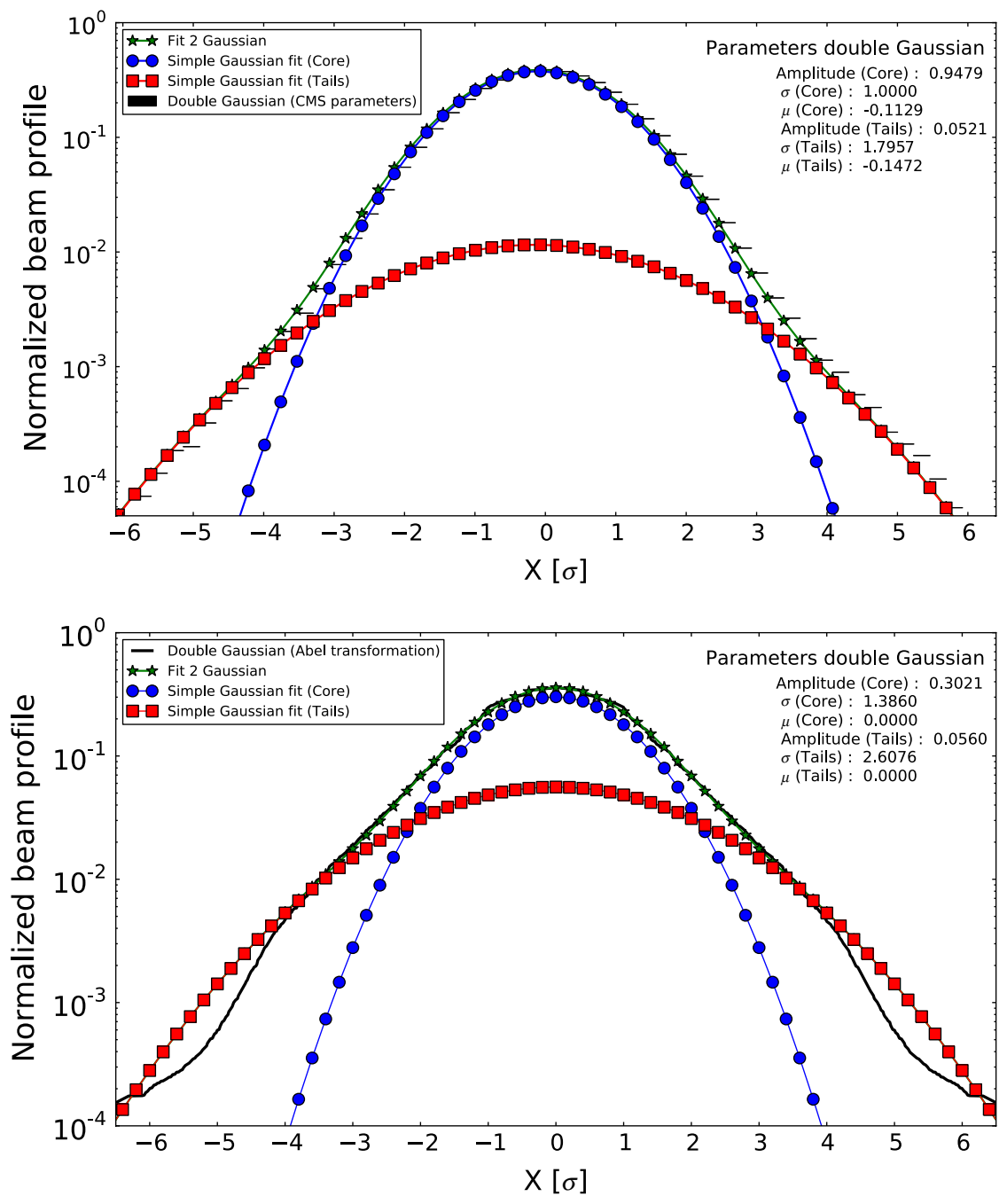

FIG. 3. The normalized horizontal beam profile extracted from data of LHC measurements. In the top, the beam distribution generated using the Van der Meer scans at CMS [28] (DG I) and in the bottom, the beam distribution obtained by applying Abel transformation to the scraping measurements [29] (DG II). 
Therefore, the formulation of this method illustrates a general approach of the luminosity as a function of the beam profile. In general the luminosity can be written as the product of the peak of the instantaneous luminosity $\left(L_{o}\right)$ by the transverse density distributions $(F(x, y))$. If the transverse distributions are uncorrelated, it is possible to express it as a product of two functions which depend only on one of the transversal variables [27,28]. In hadron machines, a Gaussian beam profile poorly describes the luminosity in the tails. A non-Gaussian profile is necessary to reproduce accurately the luminosity. A convenient way to include the contributions of the tails is by using a double Gaussian to fit the core and tails. The luminosity obtained by Van der Meer scans on CMS is fitted using a double Gaussian profile. By using the direct relation with the transversal distribution and the parameters of the fit we can obtain the horizontal beam profile $[27,28]$. The
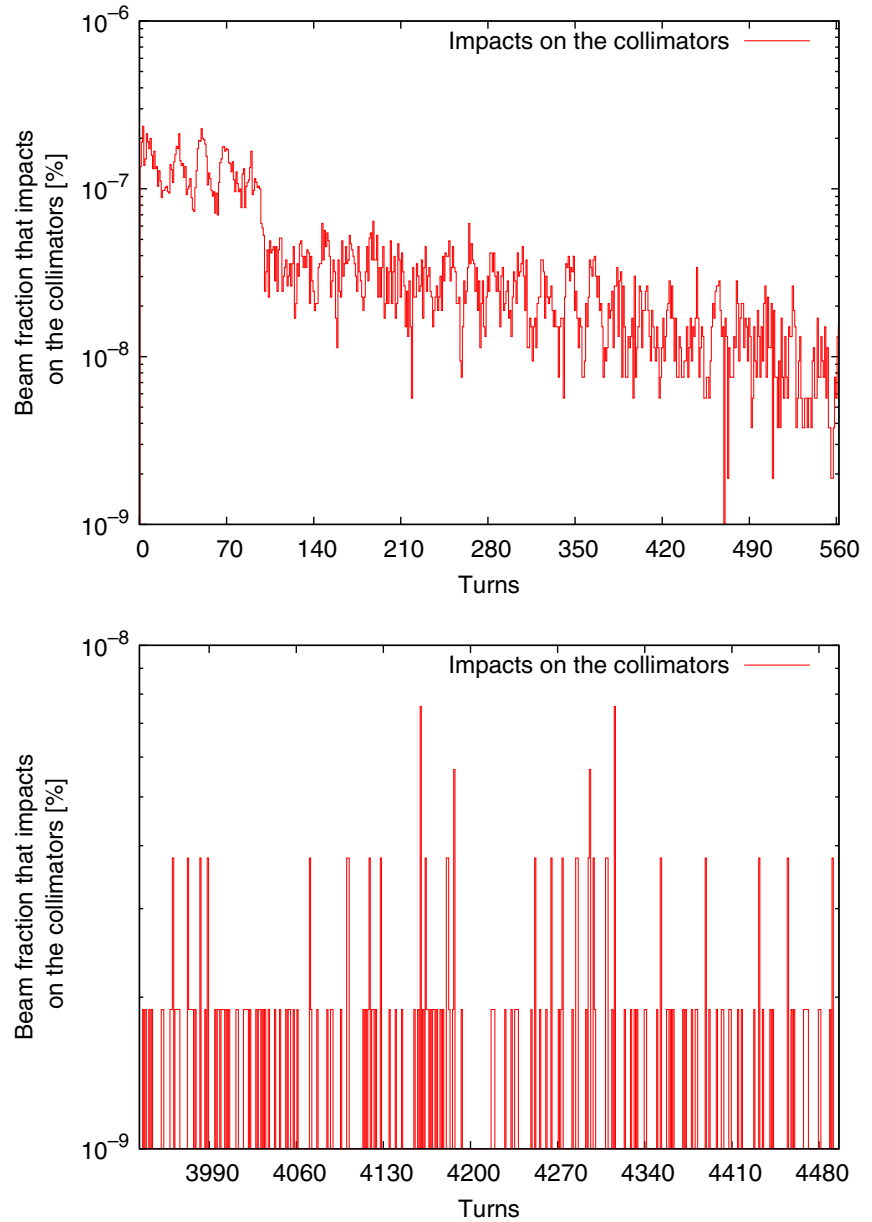

FIG. 4. Histograms of the total beam fraction that impacts on the collimators for the first and eighth synchrotron periods (562 LHC turns) to reach a quasistationary-state for beam distribution SG II. For the first synchrotron period (top) the average beam fraction that impacts on the collimators is $2.37 \times 10^{-5} \%$ and for the last synchrotron period (bottom) is $3.82 \times 10^{-7} \%$. systematic error for the luminosity is about $4.5 \%$, and given that the relation of the beam distribution and the luminosity measurements is only a factor $\left(L_{o}\right)$ we can infer that the systematics for the beam distribution are approximately the same. The percentage of the distribution beyond $4 \sigma$ is about $0.1 \%$.

(ii) DG II: During the physics operation at $450 \mathrm{GeV}$ at the LHC on July 2011, beam scraping measurements were implemented to compute beam distributions [29]. The collimation team performed slow beam scraping with a step of $40 \mu \mathrm{m}$ every $4 \mathrm{~s}$ using the TCP at the IR7. The beam loss rate recorded at different positions of the collimators generates the amplitude distribution taking into account the dispersion at the TCP for the betatron sigma. Because the beam passes several times at the collimators, the result of the scraping is a round cut. Consequently, the amplitude distribution is described by a radial function. The density distribution can be derived from the amplitude distribution through Abel transformation [26]. The
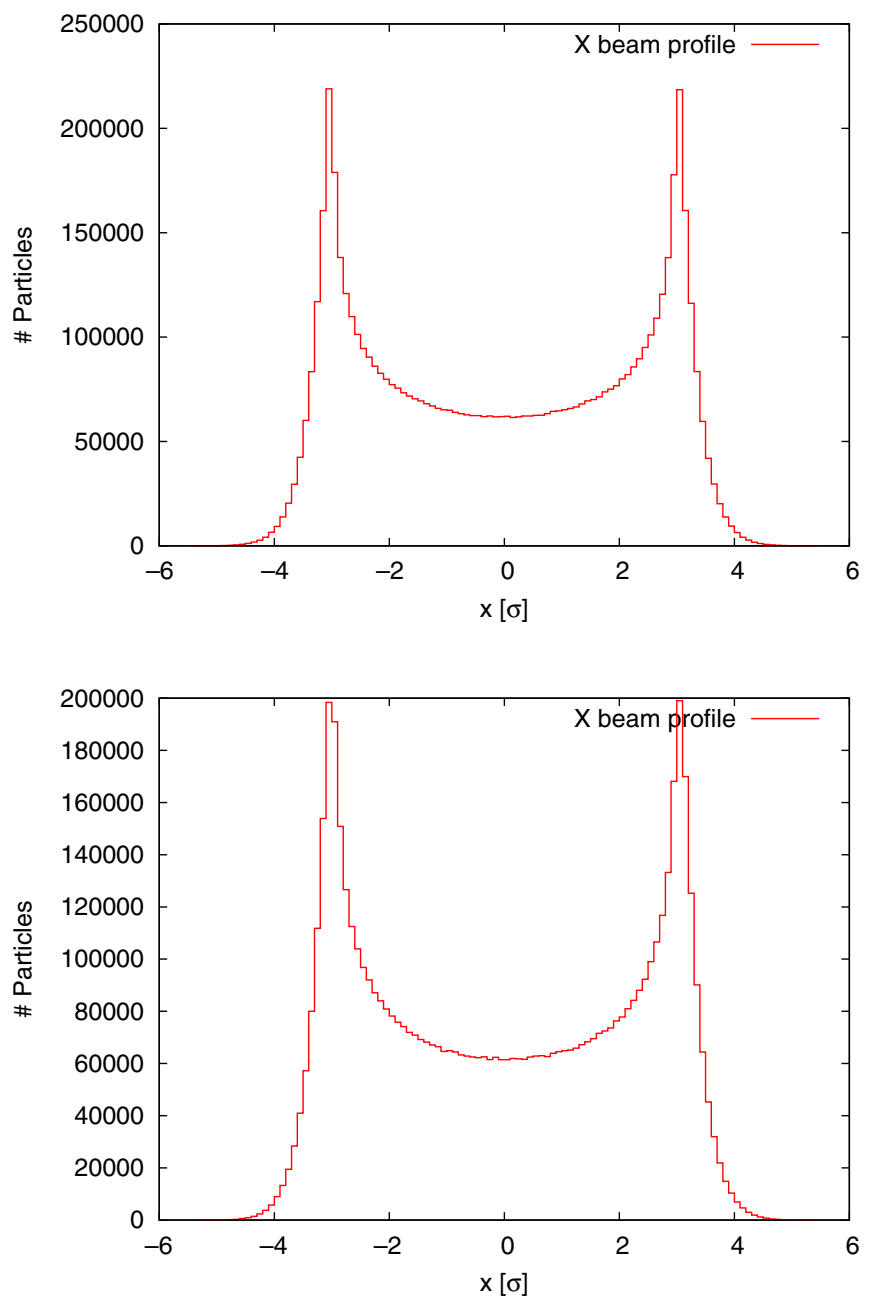

FIG. 5. Histograms of the horizontal beam distribution of SG II for the 1st turn (top) and for the 4496th turn (bottom). 
beam profile was fitted using double Gaussian for the tails, nevertheless, due to insufficient data beyond $4 \sigma$ it remains inaccurate. Using the parameters of the fit we extrapolate from $450 \mathrm{GeV}$ to $7 \mathrm{TeV}$. The beam profile is obtained assuming that the beam distribution remained static during the scraping, this is the main source of the systematics in this measurement.

To estimate the impacts for the realistic distribution, we use halos at different transverse amplitudes in the horizontal phase space with a smear of $0.1 \sigma$ ("thin-halo") and 2D Gaussian with matching beam conditions for the vertical and longitudinal phase space (similar distributions used by collimation team). Then, we obtained the approximate particle impacts on collimators and aperture for the DGs by folding these distributions with the thin-halo results (in general this works for any kind of distribution). The quality of this approximation depends on the step size of transverse amplitude in the thin-halo simulations.

All the failures are produced once the distributions reached the quasistationary-state (QSS). To obtain the QSS the
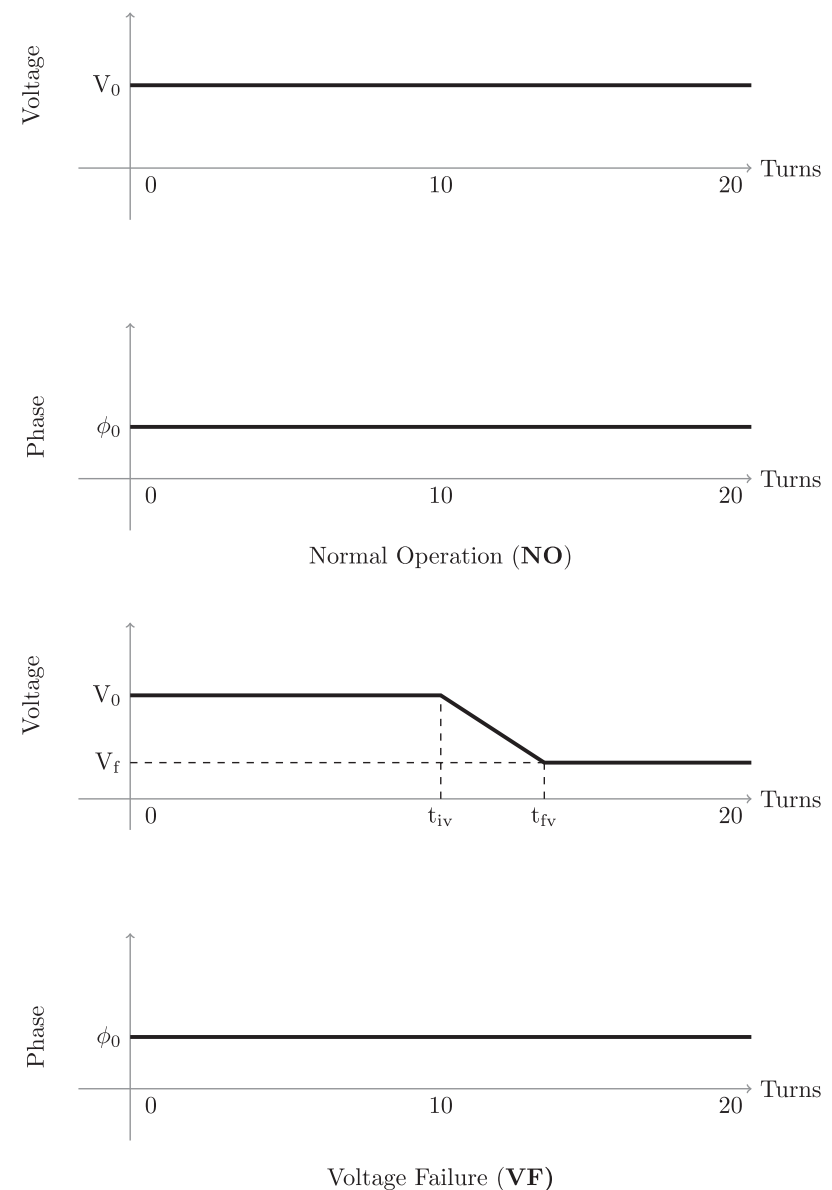

particles were tracked with and without CCs for several synchrotron periods until the beam impact rate changes slowly and becomes negligible with respect to the initial period (in average 1 particle per billion per turn). Thus, for the initial distribution of $2 \times 10^{14}$ particles in the 2012 LHC Run, the loss beam rate at the beginning of the QSS is $2.25 \times 10^{9}$ protons per second. On the other hand, for $30 \mathrm{~h}$ of exponential beam lifetime, the loss beam rate at the start of the beam filling is about $1.89 \times 10^{9}$ protons per second [30]. Therefore, our loss beam rate is close to the one that is deduced from the beam lifetime measurements at the beginning of the beam filling at the LHC in 2012. Figures 4 and 5 show examples of how a specific initial distribution with tails (SG II) reaches the QSS. This final distribution state can be considered as a realistic approximation of the steady-state (SS). The difference in the fraction of the beam impacts for tracking with and without the CCs is small $(\approx 0.4 \%)$.

In summary, for this study two different kinds of distributions were tracked until reaching the QSS before the CC failures: (1) SG distributions with Gaussian tails; (2) thin-halos which can be used to fold any beam
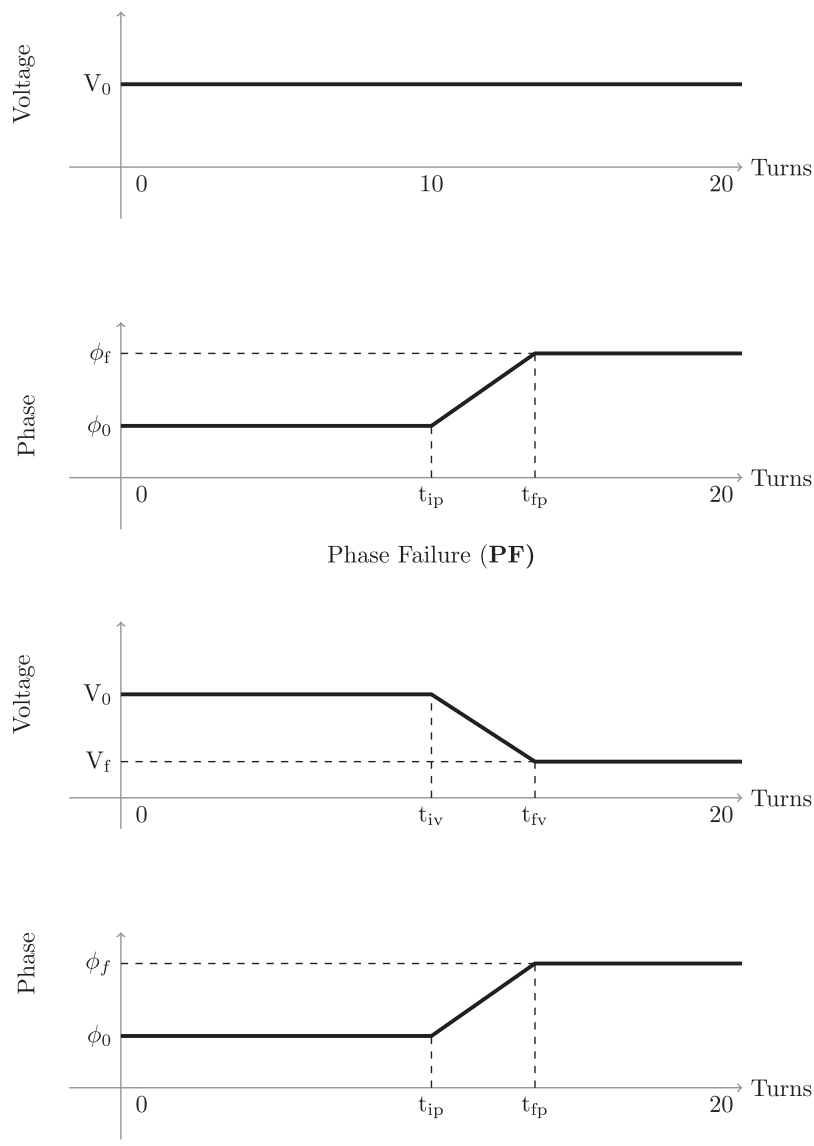

Phase Voltage Failure (PVF)

FIG. 6. Schematic ways of changing the voltage and phase through the numbers of turns during the tracking once the QSS is reached. The normal operation (NO) case (top left), the phase failure (PF) case (top right), the voltage failure (VF) case (bottom left), the phase voltage failure $(P V F)$ case (bottom right). For the PVF cases the changes start at different turns $\left(t_{i p}=t_{i v}\right)$. 
profile, in particular the two realistic distributions DG (Fig. 3).

\section{Simulated cases}

The failures are simulated by tracking two kinds of distributions. On one hand, we use the SGs defined before, on the other hand we use the thin-halos. In both schemes, after reaching the quasistationary-state, the distributions are tracked for 20 more turns and on the tenth turn the failure is initiated. The different failure scenarios considered to assess beam impacts produced by CCs are shown in Fig. 6. The normal operation (NO) case is the baseline to compare the beam impacts produced by the other cases: phase failure $(\mathrm{PF})$, voltage failure (VF), and phase voltage failure (PVF). For the PF cases, only the phase is changed and the voltage is undisturbed. Analogously, the voltage is changed and the phase is kept constant in the VF cases. Finally, in the PVF cases both the phase and voltage are changed independently.

In all the cases the voltage and the phase are varied linearly as a function of the number of turns, and the duration of the failure is 1 to 5 LHC turns, in order to simulate fast CCs failures [14-17]. The beam dump system dumps the beam in 3 LHC turns, nevertheless, the impacts were recorded for the last 10 turns after the failure. For the LHC, the natural time constant is about $800 \mu \mathrm{s}(8-10$ LHC turns) for a $\mathrm{CC}$ with $\mathrm{Q}_{\mathrm{L}}=10^{6}$ and frequency of $400 \mathrm{MHz}$. Only the last of the three CCs that closes the bump at CMS is assumed to fail. Since the CCs crab the beam in the horizontal plane at CMS, the distributions with horizontal tails are more affected by the abrupt failures.

\section{RESULTS}

For the PF and VF cases, the duration of the failures are 1,3 , and 5 LHC turns. In the PF case, we assume that $\phi_{0}$ changes from $0^{\circ}$ to $90^{\circ}$ and for the $\mathrm{VF}$, the voltage drops from nominal to zero. Finally, for the PVF cases the early change (phase or voltage) starts also on the tenth turn.

In this analysis, we ignore the initial impacts and take into account only the impacts occurring over the ten remaining turns after the failures, since the initial impacts in the quasistationary state are negligible and not related to the failure. In all the results, the beam fractions that impact are normalized to the full beam. We use two kinds of

TABLE IV. Beam fraction and the corresponding energy stored for each SG with respect to the HL-LHC full beam.

\begin{tabular}{lcc}
\hline \hline SG & \% of the beam & Energy stored $[\mathrm{kJ}]$ \\
\hline I & 97.792 & 677530.7 \\
II & 0.012 & 84.9 \\
III & 1.110 & 7621.2 \\
IV & 1.110 & 7600.4 \\
\hline \hline
\end{tabular}

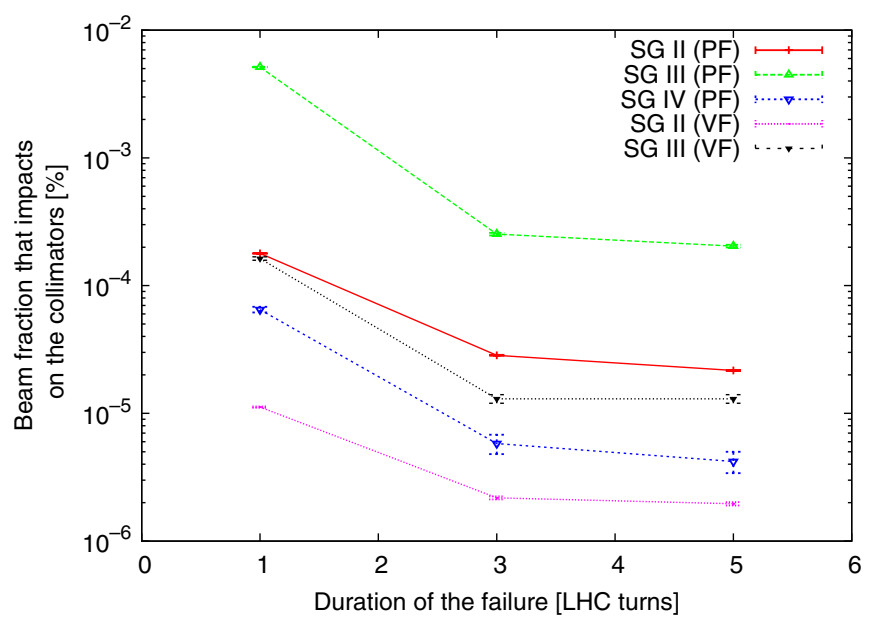

FIG. 7. Beam fraction that impacts on the collimators for the PF and VF cases considering the SG distributions. No impacts are produced in the SG IV for VF cases and in the SG I for both failure cases.

uncertainties, frequentist [31] when the numbers of event are small (impacts on the aperture) and Gaussian for the other cases. The global inefficiency is on the order of $10^{-4}$ in all the cases with and without CC failures.

The maximal beam displacement produced by the simulations for the PF and VF scenarios (in the first five turns) are around $2.1 \sigma$ and $1.6 \sigma$, respectively. Therefore, for the cases with a horizontal distribution at $2 \sigma$ below the primary collimators $(6 \sigma)$, the number of particles that impact on the collimators or on the aperture are low, or zero.

The beam percentages for the SGs and their corresponding energy are shown in Table IV.

The results for the SG distributions are shown in Figs. 7, 8 and in Table V. Only SG II registers impacts on the collimators for the NO case $\left(3.2 \pm 1.4 \times 10^{-7} \%\right.$ of the beam). The $\mathrm{CC}$ failure cases simulated with the cores in the horizontal plane produced lower beam impacts. Figure 7 shows the beam fraction that impacts on the collimators using the SG distributions for the PF and VF cases. Figure 8 displays the percentage of beam that impacts on the collimators turn by turn after the $\mathrm{CC}$ failure for SG II and SG III distributions. A periodicity of three

TABLE V. Percentage of beam that impacts on the aperture integrated over the ten remaining turns after the failures for the PF and VF cases using SG II. For SG III distribution only the PF in 1 turn case recorded beam impacts of $6.8 \pm 3.3 \times 10^{-7} \%$.

\begin{tabular}{lcc}
\hline \hline Case & Duration of the failure [turns] & Beam fraction $\left[10^{-8 \%}\right]$ \\
\hline & 1 & $3.81 \pm 0.95$ \\
$P F$ & 3 & $1.21 \pm 0.48$ \\
& 5 & $0.37 \pm 0.15$ \\
& 1 & $0.46 \pm 0.18$ \\
$V F$ & 3 & 0 \\
& 5 & 0 \\
\hline \hline
\end{tabular}





FIG. 8. Percentage of beam that impacts on the collimators turn by turn, after the failure started, for the PF (left) and VF (right) cases considering the SG II (top) and SG III (bottom) distributions.

turns on the higher peaks can be seen and it is due to the fact that fractional tunes $\left(Q_{x / y} \sim 0.3\right)$ are near to the third order resonance. These SGs produce high beam losses for the different $\mathrm{CC}$ failure cases.

In general the percentage of beam that impacts on the aperture is lower than on collimators, for instance, only the PF of 1 turn produces hits on the aperture of $6.8 \pm 3.3 \times 10^{-7} \%$ for SG III. For SG I and SG IV no impact is recorded. Table $\mathrm{V}$ shows the percentage of impacts on the aperture for the SG II distribution.

PVF cases in which the phase and voltage change simultaneously are simulated specifically, to compare with the previous cases (PF and VF for SG distributions). The final values and the duration of the failures were chosen arbitrarily. However, we have picked values less abrupt than the fast failures. Four cases are simulated:

(i) PVF I: The phase increases $10^{\circ}$ on each turn from the tenth to thirteenth turn and the voltage decays to one third of its nominal value from the twelfth to fifteenth turn.

(ii) PVF II: Similar to PVF I, the phase increases in the same way, while the voltage decays to half of its nominal value on the same number of turns. (iii) PVF III: The voltage decays to half of its nominal value from the tenth to thirteenth turn and the phase increases by $8^{\circ}$ on each turn from the eleventh to fifteenth turn.

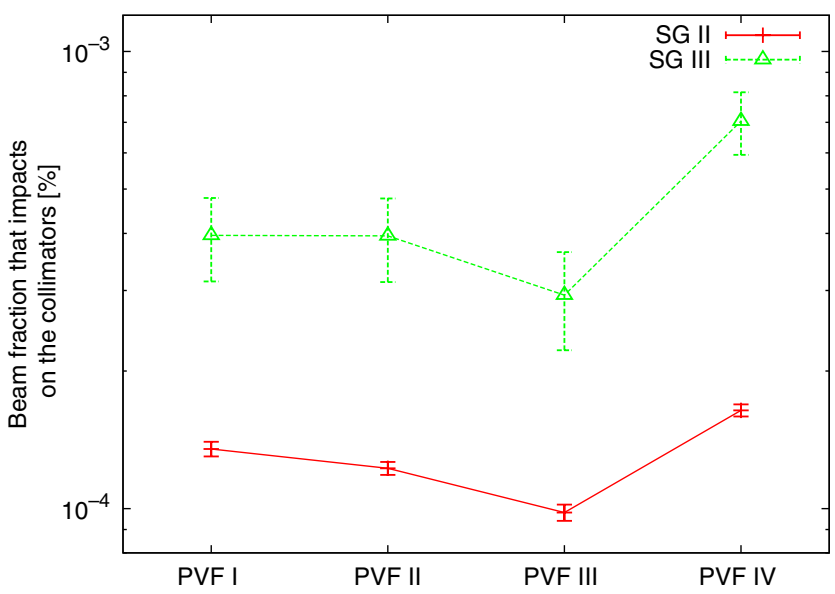

FIG. 9. Beam fraction that impacts on the collimators for the four PVF cases using the SG II and SG III distributions. 
TABLE VI. Reduction of the impacts on the collimators for the PF and VF cases using SG II and SG III by applying the mitigation strategies. In these simulations the mitigations start one turn after failure.

\begin{tabular}{|c|c|c|c|c|c|}
\hline SG & $\mathrm{CC}$ failure case & $\begin{array}{l}\text { Duration of the } \\
\text { failure [turns] }\end{array}$ & $\begin{array}{l}\text { Beam impacts without } \\
\text { mitigation }\left[\times 10^{-6} \%\right]\end{array}$ & $\begin{array}{l}\text { Beam impacts with } \\
\text { mitigation }\left[\times 10^{-6} \%\right]\end{array}$ & $\begin{array}{l}\text { Reduction of the } \\
\text { beam impacts [\%] }\end{array}$ \\
\hline & $P F$ & 1 & $178.71 \pm 0.57$ & $32.35 \pm 0.24$ & $81.89 \pm 0.14$ \\
\hline & & 3 & $28.48 \pm 0.23$ & $2.69 \pm 0.07$ & $90.52 \pm 0.26$ \\
\hline \multirow[t]{4}{*}{$S G I I$} & $V F$ & 1 & $11.19 \pm 0.14$ & $2.78 \pm 0.07$ & $75.08 \pm 0.53$ \\
\hline & & 3 & $2.18 \pm 0.06$ & $0.08 \pm 0.01$ & $95.95 \pm 0.57$ \\
\hline & $P F$ & 1 & $5136.82 \pm 29.14$ & $463.20 \pm 8.92$ & $90.98 \pm 0.16$ \\
\hline & & 3 & $253.34 \pm 6.59$ & $110.00 \pm 1.37$ & $95.65 \pm 0.53$ \\
\hline \multirow[t]{2}{*}{$S G I I I$} & $V F$ & 1 & $160.53 \pm 5.25$ & $21.31 \pm 1.91$ & $86.72 \pm 1.10$ \\
\hline & & 3 & $13.76 \pm 1.53$ & $0.34 \pm 0.24$ & $97.50 \pm 1.74$ \\
\hline
\end{tabular}

(iv) PVF IV: The change of voltage is the same as in PVF III and the phase increases by $10^{\circ}$ on each turn.

The percentage of beam that impacts for the PVF cases are shown in Fig. 9. No particle impacts on the collimators are recorded for SG I and SG IV. No particle impacts on the aperture are recorded for all SG distributions.

In addition, some mitigation strategies are implemented to quantify the possible reduction of the beam impacts for
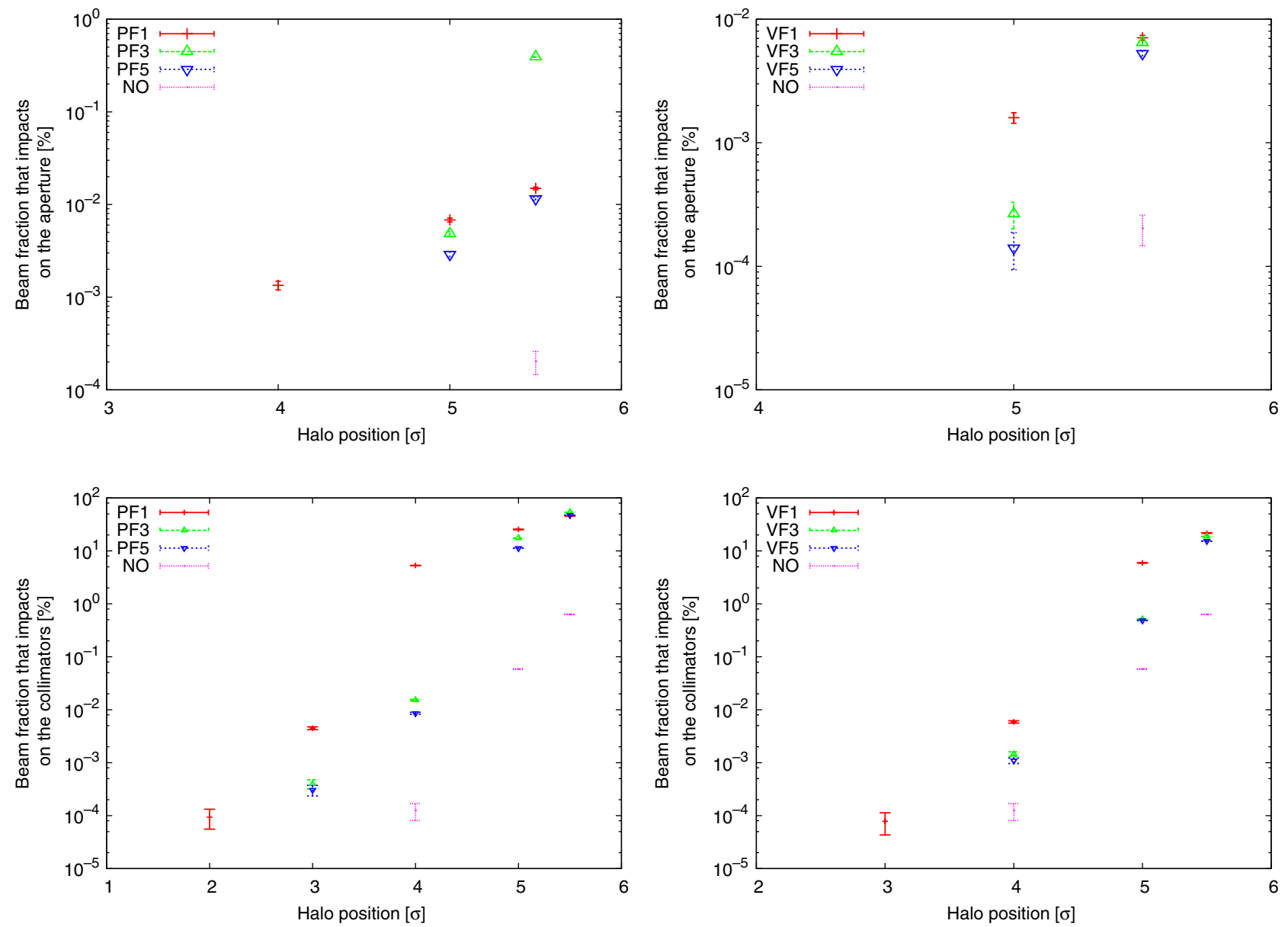

FIG. 10. Beam fraction that impacts on the aperture (top) and collimators (bottom) considering horizontal halos at different sigma positions with a smear of $0.1 \sigma$, for the PF (left), VF (right), and NO (in all the plots) cases. 

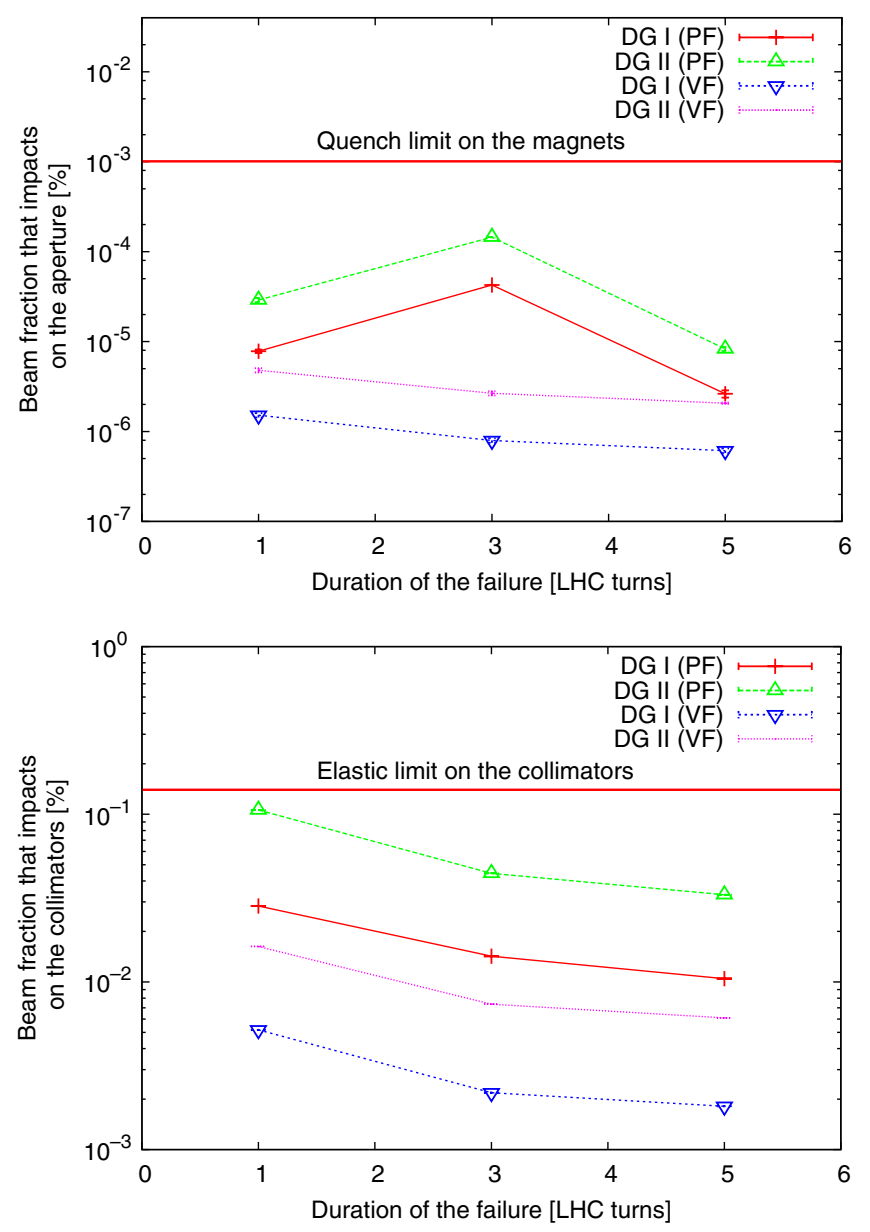

FIG. 11. Beam fraction that impacts on the aperture (top) and collimators (bottom) considering the different failure cases in voltage and phase for DG I and DG II. Errors are included.

In all the compensation cases, the voltage is dropped linearly over 3 turns. Table VI lists the percentage of beam impacts on the collimation for the cases without and with mitigation and its reduction.

Finally, in the cases of thin horizontal halo, the percentages of the beam impacts on the aperture and on the collimators are shown in Fig. 10. Using the results from the thin-halos, we roughly calculated the percentage of the beam impacts for the two realistic distributions of Fig. 3 . The percentages of the beam that impacts are shown in Fig. 11 for DG I and DG II distributions. The highest impacts are produced for the PF cases using the DG II distribution.

\section{CONCLUSIONS}

This beam tracking study shows the first results of crab cavity failures scenarios considering a realistic steady-state beam distribution in the LHC. Failures in voltage and phase were simulated for Gaussian (SG) and non-Gaussian (DG) distributions tails.
Within the proposal of the crab cavities in the LHC, one of the main concerns is its effect on the collimation system performance. The global inefficiency is $3 \times 10^{-4}$ in the normal operation of the LHC using halo at $6 \sigma$ to maximize the numbers of impacts. The values obtained using crab cavities in normal operation and in the case of failures are in the order of $10^{-4}$. Thus, the crab cavity effect on the efficiency of the collimation system is negligible.

The crab cavity produces a kick in the horizontal plane at CMS, therefore, its failure produces large losses in the horizontal distribution with long tails close to the collimators.

To estimate an upper limit for the energy deposited in our simulations, we can assume that all the particle energy is deposited on the element. Therefore, for the worst failure scenario simulated, that is phase failure in 1 turn for SG III (distribution with tails only in the horizontal plane), the energy deposited on the primary collimators is $10 \mathrm{~kJ}$ every $300 \mu \mathrm{s}$ ( 3 LHC turns) during 10 turns. In contrast, during the beam quench test in 2013, the highest acceptable load was $100 \mathrm{~kW}$ during $10 \mathrm{~s}$, that means $1 \mathrm{MJ}$. In order to compare the two results we assume that: (i) the specific heat and density of the collimators remain constant between room temperature and $150^{\circ} \mathrm{C}$ (temperature reached during the quench tests); (ii) the thermal diffusion is negligible; (iii) Fluka maps are the same; and (iv) the peak of the dynamic stress is around 2 times larger than the static one. The losses in the worst case simulated are around $6 \%$ of the elastic limit occurred during beam tests in February 2013 [32]. For the losses on the aperture the percentage of the beam that impact is $6.8 \pm 3.3 \times 10^{-7} \%$ taking the worst case (the same case that on the collimators), this value is close to the limit of the quench magnet that is $10^{-6} \%$ [12]. Nevertheless, this quench limit applies when the total beam fraction impacts only one magnet while in our simulations the losses on the aperture are deposited in several magnets. Additionally, FLUKA Monte Carlo simulations are required to evaluate the peak energy density in the coils in order to compare it with the threshold of the magnets [33].

The situation becomes worse for the two measured distributions with large non-Gaussian tails (DGs). In the most pessimistic scenario, after a phase failure in 1 turn, an amount of energy of $200 \mathrm{~kJ}$ (DG I) and $700 \mathrm{~kJ}$ (DG II) is deposited on the collimators and a beam fraction of $10^{-4} \%$ (DG I) and $10^{-3} \%$ (DG II) is deposited on the aperture during $1 \mathrm{~ms}$. This is close to the expected damage limit.

The mitigation strategies implemented, only for Gaussian tails in the horizontal plane and for failures with a duration of 1 and 3 LHC turns, show that if the crab cavity low level rf control system can react on the consecutive turns to a failure, the beam impacts may be reduced on average by $90 \%$ for the worst scenario. If the systems takes 8-10 LHC turns (natural time constant of the CCs in the LHC) to drop the voltage linearly and exponentially, the reduction is an average $71 \%$ and $65 \%$, respectively. 
In summary, exposing the LHC elements to abrupt CC failures for a period of $10 \mathrm{LHC}$ turns (3 times the normal time necessary to abort the beam) produces beam losses that are below the quench and safety thresholds for the LHC in the case of Gaussian distributions. On the contrary, for non-Gaussian distributions the quench and safety thresholds could be reached. It is noteworthy that in these simulations the population in the tails beyond $4 \sigma$ is $0.006 \%$ for SG III, $0.1 \%$ for DG I, and $0.6 \%$ for DG II, which is much lower than the overpopulated tails observed at the LHC (5\%) [29].

In previous studies [17] the energy deposited on the collimator and aperture was beyond the safety threshold for the LHC. Nevertheless, the initial conditions were totally different: the requirement of the quasistationary state before the failures was not included and the distributions had 5\% of the particle population beyond $4 \sigma$.

Halo monitoring and control during LHC operation become an essential operational tool for guaranteeing the machine safety with crab cavities operation.

\section{ACKNOWLEDGMENTS}

The authors would like to thank T. Baer, R. Bruce, F. Burkart, H. Burkhardt, F. Carra, A. Lechner, S. Redaelli, D. Wollmann, and M. Zerlauth for the comments and discussions. This project was supported by the U.S. Department of Energy through the LHC Accelerator Research Program (LARP), European FP7 HiLumi LHC Design Study, Grant Agreement No. 284404, EUCARD Acc. Net, and Conacyt México through the BEAM project and Ph.D scholarship 209889.

[1] O. Brüning, H. Burkhardt, and S. Myers, Prog. Part. Nucl. Phys. 67, 705 (2012).

[2] R. De Maria et al., in Proceedings of the 2nd International Particle Accelerator Conference, San Sebastián, Spain (EPS-AG, Spain, 2011), p. 3711; Report No. CERN-ATS2011-107, 2011.

[3] R. Palmer, Report No. SLAC-PUB-4707, 1988.

[4] K. Oide and K. Yokoya, Phys. Rev. A 40, 315 (1989).

[5] K. Oide, Prog. Theor. Phys. 122, 69 (2009).

[6] K. Nakanishi et al., in Proceedings of the International Particle Accelerator Conference, Kyoto, Japan (ICR, Kyoto, 2010), p. 2938.

[7] Y. Funakoshi, in Presentation on 4th LHC Crab Cavity Workshop, Geneva, Switzerland, 2010.

[8] G. Burt, in Presentation on 6th LHC Crab Cavity Workshop, Geneva, Switzerland, 2013; S. De Silva, in Presentation on 6th LHC Crab Cavity Workshop, Geneva, Switzerland, 2013; B. Xiao, in Presentation on 6th LHC Crab Cavity Workshop, Geneva, Switzerland, 2013.
[9] J. Wenninger, in Presentation on 4th LHC Crab Cavity Workshop, Geneva, Switzerland, 2010.

[10] O. Brüning et al., Report No. CERN-1004-003-V-1, 2004, p. 409.

[11] A. Machpherson, in Presentation on 6th LHC Crab Cavity Workshop, Geneva, Switzerland, 2013.

[12] R. Schmidt et al., in Proceedings of the 22nd Particle Accelerator Conference, PAC-2007, Albuquerque, NM (IEEE, New York, 2007), p. 878; LHC Project Report No. 1053, 2007.

[13] P. Paolo, Deduction of Steady-State Cable Quench Limits for the LHC Main Dipoles (Collimation Working Group, Geneva, Switzerland, 2013); B. Auchmann and M. Zerlauth (private communication).

[14] T. Baer et al., in Proceedings of the 24th Particle Accelerator Conference, PAC-2011, New York, 2011 (IEEE, New York, 2011), p. 76; Report No. EuCARDCON-2011-004, 2011.

[15] T. Baer et al., in Proceedings of the 2nd International Particle Accelerator Conference, San Sebastián, Spain (EPS-AG, Spain, 2011), p. 1816; Report No. EuCARDCON-2011-048, 2011.

[16] T. Baer et al., in Proceedings of the 3rd International Particle Accelerator Conference, New Orleans, LA, 2012 (IEEE, Piscataway, NJ, 2012), p. 121; Report No. CERNATS-2012-106, 2012.

[17] B. Yee-Rendon et al., in Proceedings of the 4th International Particle Accelerator Conference, IPAC2013, Shanghai, China, 2013 (JACoW, Shanghai, China, 2013), p. 906; Report No. CERN-ACC-2013-0056, 2013.

[18] Y.-P. Sun, R. Assmann, J. Barranco, R. Tomás, T. Weiler, F. Zimmermann, R. Calaga, and A. Morita, Phys. Rev. ST Accel. Beams 12, 101002 (2009).

[19] R. Calaga, in Presentation on Proceedings of Chamonix 2012 Workshop of LHC Perfomance, Chamonix, France, 2012, p. 363.

[20] L. Deniau, in Proceedings of the 11th International Computational Accelerator Physics Conference, RostockWarnemünde, Germany, 2012, p. 211; Mad-methodical accelerator design, http://mad.web.cern.ch $/ \mathrm{mad} /$.

[21] F. Schmidt, Report No. CERN/SL/94-56 (AP), 2012, http://frs.web.cern.ch/frs/six/six.html.

[22] Y.-P. Sun, R. Assmann, R. Tomás, and F. Zimmermann, Phys. Rev. ST Accel. Beams 13, 031001 (2010).

[23] A. Marsili et al., Tracking Code for Collimation Studies Extended Version of SIXTRACK for Collimation, http:// lhc-collimation-project.web.cern.ch/lhc-collimation-project/ code-tracking-2012.php.

[24] G. Valentino, R. Aßmann, R. Bruce, F. Burkart, V. Previtali, S. Redaelli, B. Salvachua, G. Stancari, and A. Valishev, Phys. Rev. ST Accel. Beams 16, 021003 (2013).

[25] S. Van der Meer, Report No. CERN-IST-PO/68-31, 1968.

[26] C. Carli et al., Part. Accel. 63, 255 (2000); Report No. CERN/PS 2000-062, 2000.

[27] S. White, Ph.D. thesis, Université Paris-Sud 11, 2010.

[28] The CMS Collaboration, Report No. CMS PAS EWK-11001, 2011.

[29] R. Assmann et al., Report No. CERN-ATS-Note-2011-057 MD(LHC), 2011. 
[30] M. Lamont, in LS1 LBOC meeting No. 11, 2013; M. LamontReport No. LHC Project Note 375 2005-1004, 2005.

[31] G. J. Feldman and R. D. Cousins, Phys. Rev. D 57, 3873 (1998).
[32] A. Bertarelli, A. Dallocchio, and T. Kurtyka, "Thermally induced vibrations of beams: Longitudinal and flexural behaviour," J. Appl. Mech. (to be published); F. Carra and $R$. Bruce (private communication).

[33] A. Lechner (private communication). 